\title{
Radon Reconstruction in Longitudinal Phase Space * REOEIVED
}

\author{
V. Mane, S. Peggs, J. Wei \\ Brookhaven National Laboratory, Upton, New York 11973, USA

\section{Abstract}

Longitudinal particle motion in circular accelerators is typically monitored by one dimensional (1-D) profiles. Adiabatic particle motion in two dimensional (2-D) phase space can be reconstructed with tomographic techniques, using 1-D profiles. A computer program RADON has been developed in $\mathrm{C}++$ to process digitized mountain range data and perform the phase space reconstruction for the AGS, and later for Relativistic Heavy Ion Collider (RHIC).

\section{INTRODUCTION}

The mathematical problem of 2D image reconstruction from an infinite number of $1 D$ projections was formulated and solved by J. Radon in 1917 [1]. In practical algorithms, now in use in computerized tomography, the image is reconstructed on a $2 \mathrm{D}$ grid from a finite number of projections. These algorithms can be broadiy classified into Fourier convolution algorithms and iterative algorithms. The Algebraic Reconstruction Technique (ART) is a commonly used iterative technique and is described by Gordon [2]. The Fourier convolution algorithm is based on papers by Bracewell and Riddle [3] and Ramachandran and Lakshminarayanan [4]. These algorithms are also presented in texts on computerized tomography [5], [6], [7].

Beam tomography in accelerators for longitudinal and transverse phase space has been used by several authors [8], [9], [10], using the ART algorithm. In longitudinal phase space, the synchrotron osillation period is typically several hundred turns and many bunch profiles are available over half a period. Therefore the Fourier convolution techniques are adequate. In this paper, we present reconstruction for mountain range data shown in Figure 1 and discuss a filtered backprojection algorithm for phase space reconstruction. During the Sextant test of RHIC in early 1997 , this program was employed to reconstruct the motion of $\mathrm{Au}^{77+}$ beam in the AGS.

\section{RADON TRANSFORM AND RECONSTRUCTION}

Let $f(x, y)$ represent a $2 D$ object (Figure 2). Let $\left(x_{r}, y_{r}\right)$ be the coordinate system rotated by angle $\theta$ with respect to the $(x, y)$ coordinate system, where $x_{r}=x \cos \theta+y \sin \theta$ and $y_{r}=-x \sin \theta+y \cos \theta$. The projections $P\left(x_{r}, \theta\right)$ obtained by integrating $f(x, y)$ along all lines of constant

\footnotetext{
- Work performed under the auspices of the U.S. Department of Energy.
}

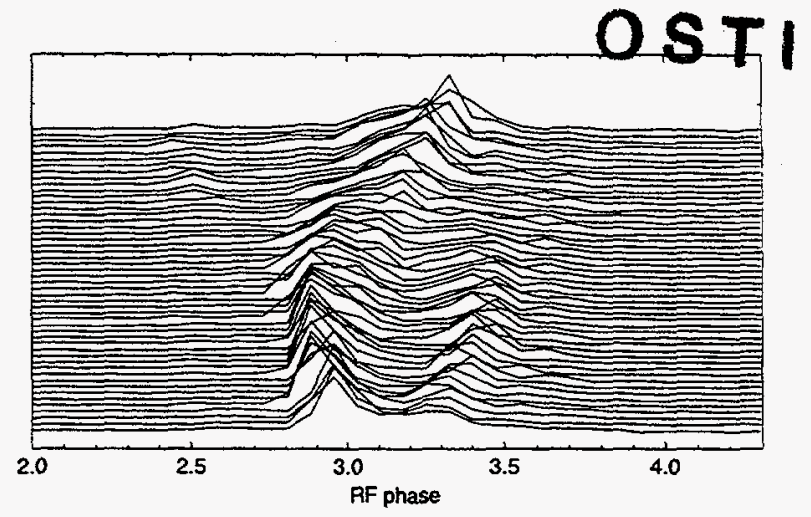

Figure 1: Mountain Range Data from the AGS, used for reconstruction in figure 5 .

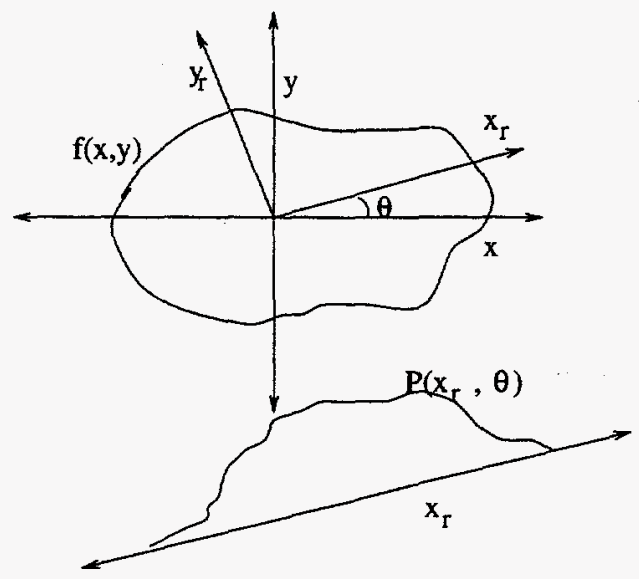

Figure 2: The profile $P\left(x_{r}, \theta\right)$ is the Radon Transform of the two dimensional function $f(x, y)$.

$x_{r}$ in the plane is the Radon transform of $\mathrm{f}(\mathrm{x}, \mathrm{y})$

$$
P\left(x_{r}, \theta\right)=\int_{-\infty}^{\infty} f\left(x_{r}, y_{r}\right) d y_{r}
$$

The filtered backprojection algorithm, a form of Fourier convolution algorithm, is employed in the program RADON. In this algorithm, each projection $P\left(x_{r}, \theta\right)$ is Fourier transformed, multiplied by a high pass filter, inverse Fourier transformed, backprojected, and all the backprojections are summed up. Note that backprojection is not the inverse of projection, and hence the high pass filter.

Let $F(u, v)$ be the 2D Fourier Transform of $f(x, y)$ and let $(w, \theta)$ represent the polar coordinates in the frequency space, with $u=w \cos \theta$ and $v=w \sin \theta$

$$
\begin{aligned}
f(x, y) & =\int_{-\infty}^{\infty} \int_{-\infty}^{\infty} F(u, v) e^{i 2 \pi(u x+v y)} d u d v \\
& =\int_{0}^{2 \pi} \int_{0}^{\infty} F(w, \theta) e^{i 2 \pi w(x \cos \theta+y \sin \theta)} w d w d \theta(2)
\end{aligned}
$$


Since $x_{r}=x \cos \theta+y \sin \theta$ and $F(w, \theta+\pi)=F(-w, \theta)$, the above equation can be written as

$$
f(x, y)=\int_{0}^{\pi} \int_{-\infty}^{\infty} F(w, \theta)|w| e^{i 2 \pi w x_{r}} d w d \theta
$$

The Fourier Transform of a projection at angle $\theta$ with respect to $x$ gives a slice of the 2D Fourier Transform $F(u, v)$ at angle $\theta$ wrt $\mathrm{u}$. Therefore, $F(w, \theta)$ is the Fourier Transform of $P\left(x_{r}, \theta\right)$ and

$$
Q\left(x_{r}, \theta\right)=\int_{-\infty}^{\infty} F(w, \theta)|w| e^{i 2 \pi w x_{r}} d w
$$

gives the filtered projection, where the frequency response of the filter is given by $|w|$. The reconstructed image can be obtained by integrating over the filtered backprojections

$$
f(x, y)=\int_{0}^{\pi} Q\left(x_{r}, \theta\right) d \theta
$$

The following two filters are defined in RADON [6] Ramp Filter

$$
H(f)= \begin{cases}|f| & \text { if }|f| \leq f_{c} \\ 0 & \text { otherwise }\end{cases}
$$

where $f_{c}$ is the cutoff frequency

Hann Filter

$$
H(f)= \begin{cases}0.5|f|\left(1+\cos \left(\pi f / f_{c}\right)\right) & \text { if }|f| \leq f_{c} \\ 0 & \text { otherwise }\end{cases}
$$

\section{RECONSTRUCTION EXAMPLES}

The program RADON, written in $\mathrm{C}++$ generates simulation profiles, reconstructs images, and displays them graphically. The program also reads mountain range data, reconstructs in longitudinal phase space, and calculates the longitudinal emittance. This section gives an example of image reconstruction from simulation and compares it with the original image. Reconstruction with data acquired from AGS is described in next section. The signal and background errors, as described below, compare the original image with the reconstruction.

\subsection{Signal Error}

Let $I_{i j}$ and $X_{i j}$ be the intensity of the original image and the reconstructed image, respectively, in cell $(i, j)$. Let $I_{\max }$ be the maximum intensity of the original image. Define the cutoff intensity $I_{c}$ to be $10 \%$ of the maximum intensity, that is $I_{c}=0.1 I_{m a x}$. Define the signal region $\mathrm{S}$ such that $I_{i j}>I_{c}$. Signal Error is defined by

$$
E_{s}=\frac{1}{I_{\max }-I_{\min }} \sqrt{\frac{1}{\alpha_{s}} \sum_{i, j \in S}\left(I_{i j}-X_{i j}\right)^{2}}
$$

where $\alpha_{s}=\sum_{i, j \in S} 1$

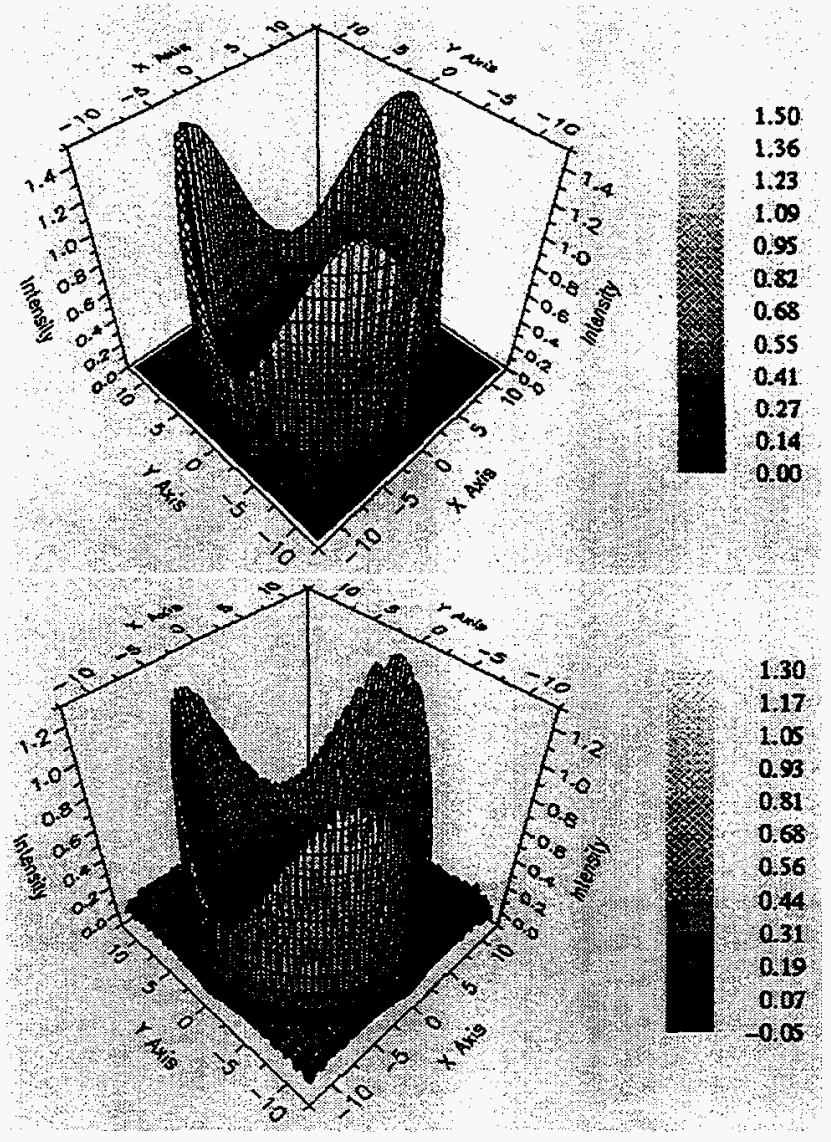

Figure 3: Original image (top) and reconstructed image using RADON (bottom). A total of 30 profiles with 64 samples per profile are used for reconstruction.

\subsection{Background Error}

Define the background region $B$ consisting of all the cells $(i, j)$ that are not in S. Background Error is defined by

$$
E_{b}=\frac{1}{I_{\max }-I_{\min }} \sqrt{\frac{1}{\alpha_{b}} \sum_{i, j \in B}\left(I_{i j}-X_{i j}\right)^{2}}
$$

where $\alpha_{b}=\sum_{i, j \in B} 1$

\subsection{Example}

Figure 3 compares the reconstructed image with the original image for an annular distribution, modulated in the azimuthal direction. A total of 30 profiles equally spaced between 0 and $\pi$, with 64 samples per profile are used for the reconstruction. The signal and background errors are 0.21 and 0.07 , respectively. In this case, the signal error is due to the difference in amplitude of the reconstruction. Sensitivity analysis were performed to determine the error in reconstruction due to insufficient profiles, samples and the error in synchrotron period. Figure 4 gives the signal and the background errors, for a gaussian distribution due to the error in synchrotron period. Several examples of reconstruction with gaussian and annular distribution, and the associated errors are given in [11]. 


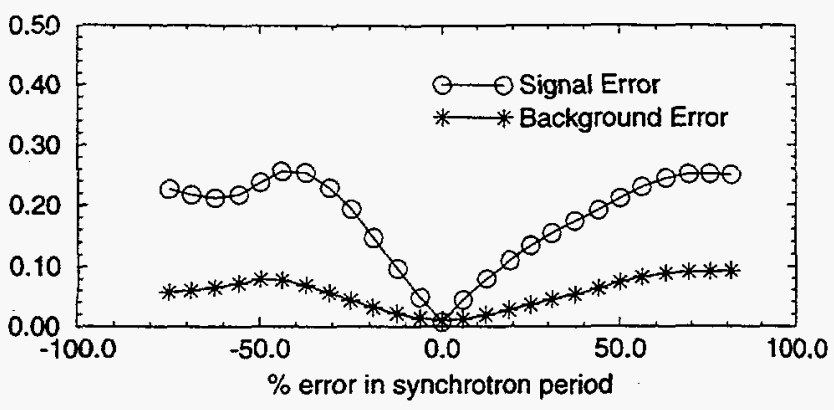

Figure 4: Signal and Background error vs. error in synchrotron period, for a gauss beam.

\section{APPLICATION IN RHIC SEXTANT TEST}

RADON application was successfully used during the Spring 1997 RHIC Sextant Test [12] for longitudinal phasespace reconstruction of $\mathrm{Au}^{77+}$ beams in AGS. Mountainrange beam profile data from the Wall Current Monitor taken at AGS exrtraction flattop was captured into a fast scope, LeCroy Model 9354TM, with a rearm time of 100 $\mu s$. The data was transfered to a computer via GPIB using a LabVIEW program.

Particle motion was reconstructed from a set of profiles recorded over half a synchrotron period (Figure 1), along with basic parameters of the machine and the rf system. Since the bunch only occupied a small fraction of the rf bucket, synchrotron motion was linearized. The rf voltage was calibrated by observing the synchronous phase difference as transition was crossed at a known ramp rate. Signal width broadening caused by finite cable bandwidth [12] was taken into account.

Figure 5 shows the reconstructed phase space diagram at an early stage of the test after two bunches were coalesced to increase the beam intensity. The bunch rotated by $110^{\circ}$ in synchrotron motion, from the top figure to the bottom figure. The calibrated rf voltage was $254 \mathrm{kV}$, the harmonic number was 8 , the rf frequency was $2.96 \mathrm{MHz}$, the relativistic $\gamma$ was 12.1 , transition $\gamma_{t}$ was 8.49 , and the synchrotron period was $9.6 \mathrm{~ms}$. Totally, 49 profiles with $100 \mu \mathrm{s}$ separation sampled at $4 \mathrm{~ns}$ were used for the reconstruction.

\section{CONCLUSIONS}

A program RADON has been developed to reconstruct 2D longitudinal phase space plot of the beam from 1D profiles. During the Sextant test of RHIC in early 1997, this program has been successfully employed to reconstruct the motion of $\mathrm{Au}^{77+}$ beam in the AGS. In the future, we plan to generalize the application for both longitudinal and transverse phase space, including nonlinear motion.

\section{ACKNOWLEDGEMENTS}

We would like to thank W. VanAsselt for the LabVIEW MountainRange program, and for his help with obtaining data. We would also like to thank S. Tepikian for the XRT
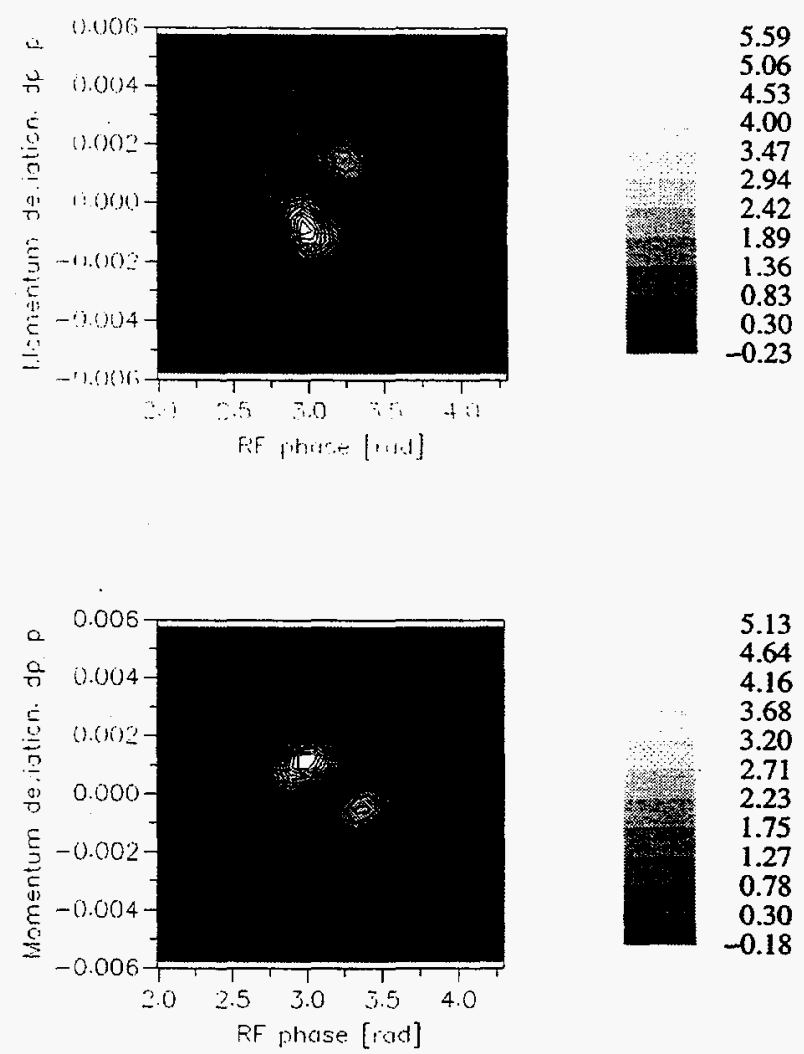

Figure 5: This figure shows two distinct globs of charge of $\mathrm{Au}^{77+}$ rotating in an AGS $\mathrm{rf}$ bucket, with the bottom frame rotated by 110 degree wrt the top frame.

graphics display program, B. Andrews, $\mathrm{K}$. Brown and the AGS Main Control Room crew for their help and support.

\section{REFERENCES}

[1] Johann Radon, Ber. Verh. Sächs. Akad. Wiss. Leipzig, Math. Phys. K1. 69, 262-277 (1917).

[2] R. Gordon, R. Bender, G. T. Herman, J. Theor. Biol., (1970), $29, \mathrm{pp} 471-481$.

[3] R. N. Bracewell, A. C. Riddle, Astrophys. J. 150 (2), pp 427434 (1967).

[4] G. N. Ramachandran, A. V. Lakshminarayanan, Proc. Natl. Acad. Sci. 68 (9), pp 2236-2240 (1971).

[5] Stanley R. Deans, The Radon Transform and some of its applications, J. Wiley, 1983.

[6] G. T. Herman, Image Reconstruction from Projections, Springer-Verlag, 1979.

[7] A. Kak, M. Slaney, Computerized Tomographic Imaging, IEEE Press, 1988.

[8] J. S. Fraser, IEEE Trans. Nucl. Sci., Vol. NS-26, Feb 1979, pp 1641-1646.

[9] O. R. Sander et al., BNL-51 134 (1980), 314.

[10] G. Jackson, IEEE PAC, Washington D. C. (1993), 2418.

[11] V. Mane, RHIC/AP/96, June 1996.

[12] J. Wei et al., "RHIC Sextant Test - Accelerator Systems and Performance", these proceedings. 


\section{DISCLAIMER}

This report was prepared as an account of work sponsored by an agency of the United States Government. Neither the United States Government nor any agency thereof, nor any of their empleyees, makes any warranty, express or implied, or assumes any legal liability or responsibility for the accuracy, completeness, or usefulness of any information, apparatus, product, or process disclosed, or represents that its use would not infringe privately owned rights. Reference herein to any specific commercial product, process, or service by trade name, trademark, manufacturer, or otherwise does not necessarily constitute or imply its endorsement, recommendation, or favoring by the United States Government or any agency thereof. The views and opinions of authors expressed herein do not necessarily state or reflect those of the United States Government or any agency thereof. 


\section{DISCLAMMER}

Portions of this document may be illegible in electronic image products. Images are produced from the best available original document. 